\title{
Correlation of Cryo Soft X-ray Tomography with Cryo Fluorescence Microscopy to Characterise Cellular Organelles at Beamline B24, Diamond Light Source
}

Matthew C. Spink $^{1} *$, Michele C. Darrow ${ }^{1}$, Hannah Fisher ${ }^{1}$, Alister Burt ${ }^{1}$, Karen Marshall ${ }^{2}$, Imanol Luengo $^{1}$, Maria Harkiolaki ${ }^{1}$, Liz Duke ${ }^{1}$.

${ }^{1}$ Diamond Light Source, Harwell Science and Innovation Campus, Chilton, Didcot, OX110DE. UK

${ }^{2}$ University of Sussex, Department of Biochemistry and Molecular Biology, Brighton, BN1 9RH

* Corresponding author, matthew.spink@diamond.ac.uk

Introduction Cryo soft-X-ray tomography (Cryo-SXT) is a 3D imaging technique that allows us to image whole vitrified biological cells and their contents in a near native state. Cryo-SXT utilises the natural contrast of cellular structures by imaging in an energy range termed the 'water window' The water window is between the $\mathrm{K}$ absorption edges of Carbon $284 \mathrm{eV}$ and Oxygen $543 \mathrm{eV}$ [1]. Imaging between these energies results in higher X-ray absorption by carbon rich cellular structures than oxygen containing molecules such as vitrified water [1]; [2]. The resulting contrast allows us to see cellular structures (Figure 1A) at a resolution of $\sim 40 \mathrm{~nm}$, depending on the sample and microscope components [3].

Often, in order to answer biological questions using cryo-SXT cellular organelles need to be chemically identified. To address this, cells are fluorescently labelled for specific organelles and imaged using fluorescent microscopy (Figure 1B). Correlation of fluorescence with cryo-SXT volumes allows for the identification of specific organelles within the 3D context of the cell. Identifying specific organelles in cryo-SXT volumes provides the potential of being able build up profiles for different cellular organelles.
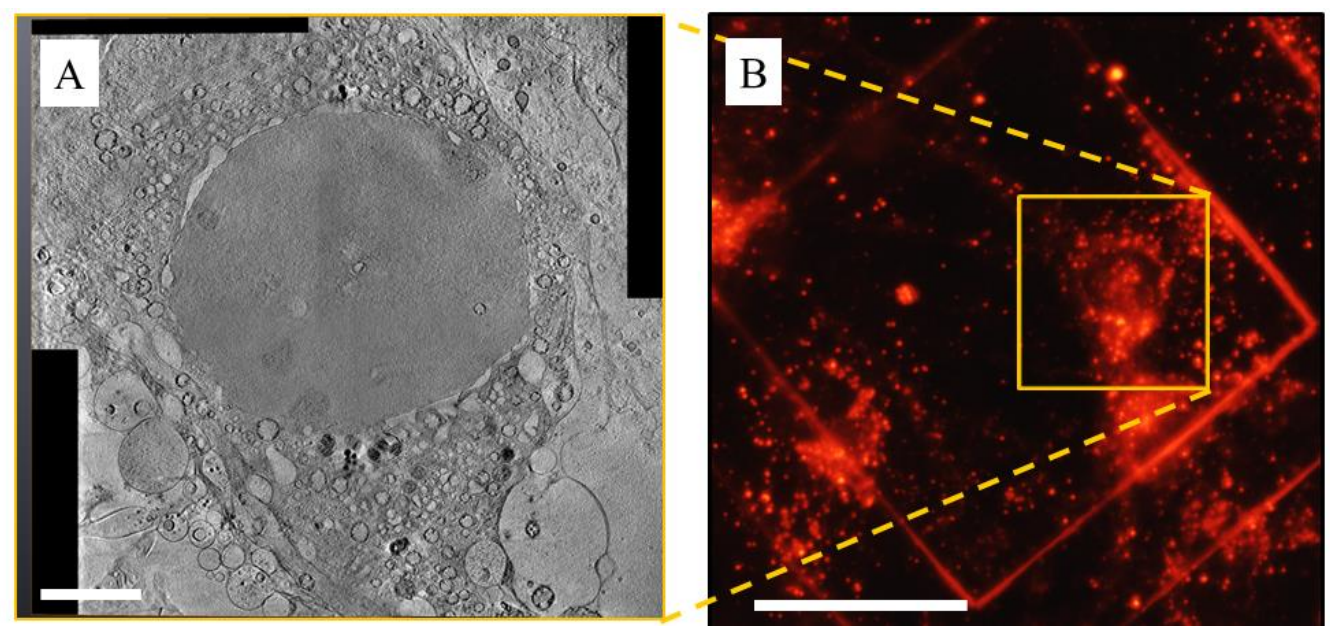

Figure 1. A) Example dataset from cryo-SXT, image is composed of four overlapping tilt series of a primary neuron; B) Example of fluorescence microscopy of the same neuron fluorescently labelled for lysosomes. Scale bar A=5 $\mathrm{m} ; \mathrm{B}=50 \mu \mathrm{m}$ Sample and datasets provided by Karen Marshall and Louise Serpell, University of Sussex.

Experimental procedures Sample preparation; Primary neurons (Figure 1) or HEK293 (Figure 2) cells were grown grids with a holey carbon substrate [4], cells were fluorescently labelled for lysosomes or late endosomes. Fiducials were added, the grid was then blotted and plunged into liquid ethane. 
Imaging; Cryo-SXT was performed using a Zeiss (previously Xradia) soft X-ray microscope (UltraXRM-S/L220c) at 500ev X-rays. Tomographic reconstruction was performed using the Imod package [5] Cryo-SXT volume stitching was performed using Avizo/Amira (FEI). 2D epifluorescence imaging (Figure 1B) was performed using a Zeiss Axio Imager M2 fitted with a Linkam cryostage (CMS196). 3D fluorescence imaging (Figure 2A) was performed on the B24 cryo-SIM microscope (system resolution $\sim 245 \mathrm{~nm}$ ). Volume correlation was done using ICY-ecCLEM [6] and segmentation was performed using SuRVoS [7].

\section{Main results and conclusions}

Using Cryo-SXT we have collected data on HEK 293 cells (an established mammalian cell line. We have treated cells with a number of commercially available fluorescent probes which localise to lysosomes and late endosomes and imaged them using cryo-SIM. The 3D volumes of fluorescence and cryo-SXT have been combined to identify organelles within the context of the cells (Figure 2A). Using this correlation specific organelles have been segmented out from the cryo-SXT volume (Figure 2B) using a segmentation software called SuRVoS [7].
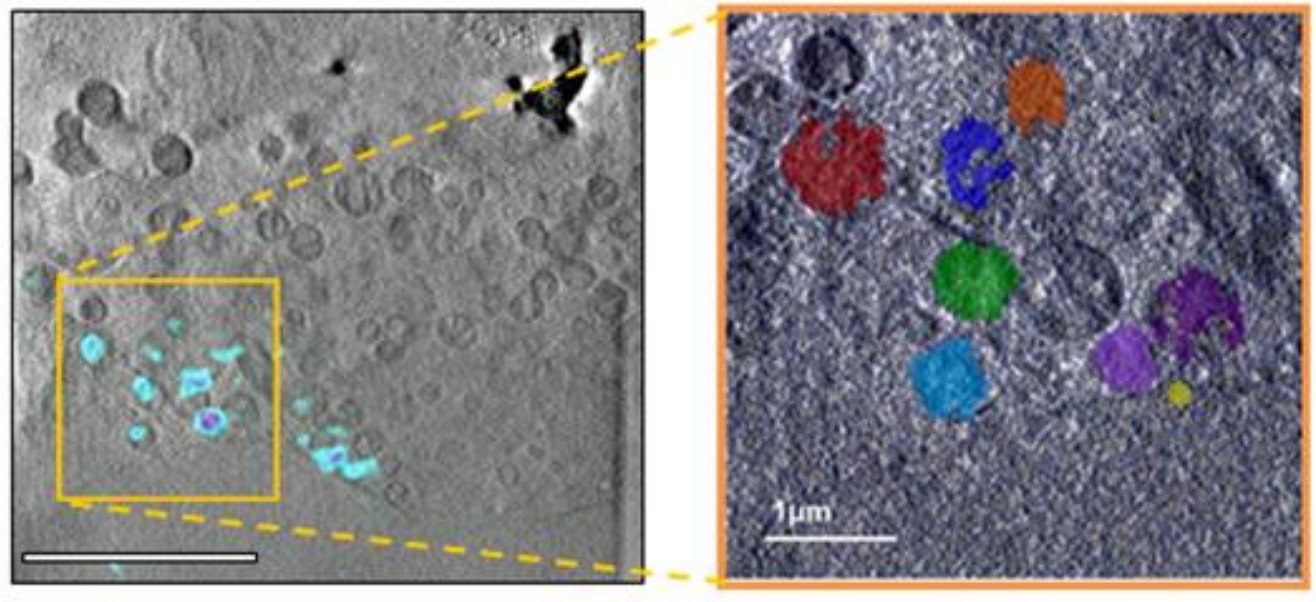

Figure 2.

A) A section through the correlated CryoSXT and fluorescence of late endosomes volumes of HEK293 cells; B) Segmentation of late endosomes in Cryo-SXT volume based on correlative fluorescence information. Scale bar $\mathrm{A}=6 \mu \mathrm{m} ; \mathrm{B}=1 \mu \mathrm{m}$

Future work will involve analysing each type of segmented organelles in order to build up profiles. This will be done on multiple organelles and in different cell lines. If sufficient profiles for organelles are developed this may reduce the need for fluorescently labelling samples as the segmentation could be classified based on organelle profiles. Additionally the workflow of correlating cryo-SXT and fluorescence volumes as demonstrated here will be used in other projects to provide insight into biological samples.

\section{References}

[1] G.Schneider et al, Journal of Structural Biology, 177 (2012), 212-223.

[2] R Carzaniga, M-C. Domart, E.Duke, L.M. Collinson, Methods in Cell Biology, 124 (2014), 151-178.

[3] R. Carzaniga, M.-C. Domart, L.M.Collinson ,E.Duke, Protoplasma, 251 (2014), 449-458.

[4] M. Harkiolaki et al, Emerging Topics in Life Sciences, In press.

[5] J.R. Kremer, D,N. Mastronade, J.R. Mcintosh, Journal of Structural Biology, 116 (1996), 71-76.

[6] P.Gilloteaux et al, Nature Methods, 102 (2017), 102-103.

[7] I. Luengo et al, Journal of Structural Biology, 198 (2017), 43-53. 